\title{
TECNOLOGÍA QUE EVITA LA REVICTIMIZACIÓN EN NIÑOS, NIÑAS Y ADOLESCENTES: CÁMARA GESELL Technology that prevents re-victimization in children and adolescents: Camera Gesell
}

\author{
Elizabeth Consuelo Ulfe Herrera ${ }^{1}$
}

Ministerio Público, Perú

Recibido: 2-11-2015

Aceptado: 27-12-2015

\section{RESUMEN}

A diario los medios de prensa publican y difunden noticias impactantes relacionadas con el abuso sexual, siendo los niños las víctimas más indefensas de tan execrable crimen. En Latinoamérica, Perú registra la mayor tasa de denuncias por abuso sexual, siendo el tercer país a nivel mundial con mayores índices de violación a menores. Ante los hechos, las autoridades intentan encontrar una solución eficaz para que los niños abusados no sufran daños adicionales a los que ya padecieron a manos de sus agresores, es decir, se trata de evitar la revictimización; para ello aprovecha los recursos de la Tecnología. Una de éstas tecnologías, iniciada entre 1940 y 1950 por el Dr. Arnold Gesell y que ha sido mejorada e implementada en algunos países como, Honduras, Argentina, Bolivia, Perú entre otros, es la denominada cámara Gesell, resulta ser una posible solución al problema de la revictimización. El uso de esta tecnología y su implicancia forma parte del análisis bibliográfico que se realiza en este artículo, describiendo las ventajas que tiene frente al método clásico de tomar a diario declaraciones cara a cara con las víctimas. El presente documento, es el resultado de una investigación bibliográfica en las base de datos de ciencias sociales, como CSIC, ISOC-Derecho, E-Journal, Clacso.Redalyc. org y Dialnet, el objetivo fue identificar información actualizada acerca del uso de la cámara de Gesell, considerada como estrategia eficaz para evitar la revictimizacion de niño, niña o adolescente.

Palabras Clave: Abuso sexual, revictimización, cámara de Gesell, tecnologías.

\section{Abstract}

Every day the media publishes and releases shocking news related to sexual abuse, being the children the most helpless victims of this execrable crime. In Latin America, Peru has the highest rate of complaints regarding sexual abuse, being the third country in the world with the highest rates of child assault. Given the facts, the authorities try to find an effective solution for abused children so that they don't suffer additional damage to those that they have already suffered at the hands of their attackers; in other words, they try to avoid revictimization; in order to do that, resources of technology are being utilized. One of these technologies, launched between 1940 and 1950 by Dr. Arnold Gesell , which has been improved and implemented in some countries, such as Honduras,

1 Abogada, candidata a magister por la Universidad Nacional Mayor de San Marcos, Fiscal Provincial de Familia de Lima, Distrito Judicial de Lima, ex Juez de la Corte Superior del Callao, Segunda Especialidad en Psicoterapia Familiar Sistémica UNFV, egresada de la Facultad de Psicología de la Universidad Alas Peruanas, ha participado en cursos sobre el nuevo modelo procesal en Chile, Colombia, California y Puerto Rico, TIC en Costa Rica, Curso Avanzado sobre el Proyecto del Código Civil UBA Argentina; eulfe@mpfn.gob.pe. 
Argentina, Bolivia, Peru, among others, is the Gesell Camera, which turns out to be a possible solution to the problem of revictimization. The use of this technology and its implications is part of the bibliographic analysis undertaken in this article, describing its advantages compared to the classical method of taking statements daily face to face with the victims. This document is the result of a bibliographic research in social science databases, such as CSIC, ISOC-Derecho, E-Journal, Clacso, Redalyc.org and Dialnet. The objective was to identify updated information about the use of the Gesell Chamber, considered as an effective strategy to prevent the revictimization of the child or adolescent

Keywords: Innovation, Technology, Entrepreneurship, Technological Centers.

\section{INTRODUCCIÓN}

En los últimos cuatro años, la tasa de delitos sexuales en agravio de niños, niñas y mujeres adultas se ha incrementado de un 46\% a 52\% (Sierra, 2013). En Sudamérica (figura 1), Perú registra la tasa más alta de denuncias por violación sexual (22.4\%). Esta es una situación alarmante que debe tomarse en cuenta en el plano jurídico y político si se quiere dar solución al problema. Los datos específicos que se muestran en la figura 2 respecto a agresores y víctimas, muestran que en el año 2013 se reportaron diariamente 69 denuncias por violación sexual.

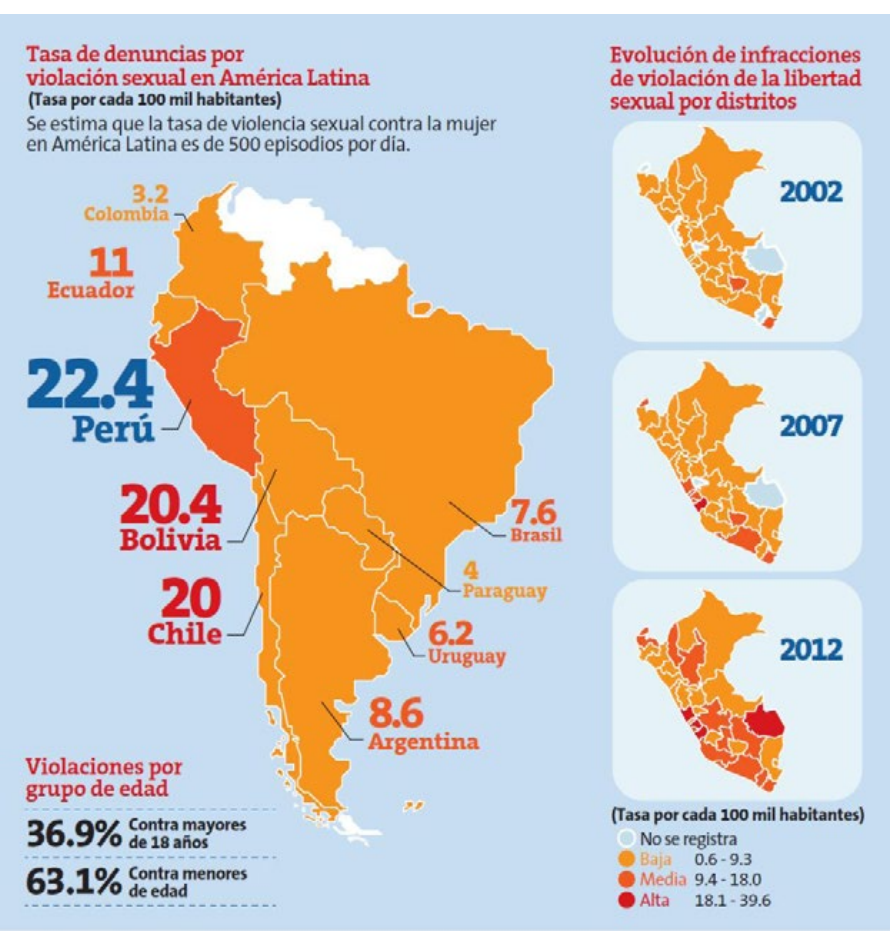

Figura 1. La tasa de denuncias por violación sexual en Latinoamérica, y en Perú a través de los últimos 13 años. Fuente: http://peru21.pe/multimedia/imagen/t-252944
El delito contra la libertad sexual en agravio de niños, niñas y adolescentes genera una evidente y profunda afectación a su integridad física, espiritual o psicológica, como consecuencia de los episodios traumáticos vividos y termina afectando su personalidad y la manera cómo se relacionarán con otros individuos en el futuro (Paredes, s.f.). Estos delitos ya cuentan con un marco jurídico amplio para la defensa del agraviado (Peláez, 2010), entre estos están: la Constitución Política del Estado, la Convención sobre los Derechos del Niño, la Declaración de Principios Fundamentales de Justicia para las Víctimas de Delitos y del Abuso de Poder, el Código de los Niños y Adolescentes, el Código Penal y normas conexas, el Código de Procedimientos Penales, el Código Procesal Penal, la Ley que establece la acción penal pública en los delitos contra la libertad sexual (Ley N²7115), la Ley General de Salud y el Plan Nacional de Acción por la Infancia y Adolescencia, constituyen un cuerpo normativo que garantiza la protección e integridad de niños, niñas y adolescentes.

Sin embargo, además de las normas mencionadas anteriormente, existe un método de atención clásico al afectado de abuso, en donde se procede por medio de representantes de la ley, que pueden o no tener experiencia en estos casos, que toman la información brindado por el niño sobre el evento sucedido, además es necesario un seguimiento de varios días debido a que se necesita completar toda la información posible sobre este suceso, de modo tal que hace el niño tenga que recordar ese evento tan nefasto para él. (Bagnasco, 2015)

A pesar de esto, hay otras alternativas más actuales 
para evitar que un niño, niña o adolescente abusado sea otra vez víctima de estupro y no pase por el daño psicológico de recordar por varios días ese acontecimiento. La sala de entrevista-cámara de Gesell constituye un espacio dotado de alta tecnología, en el cual la víctima es entrevistada y examinada de la manera más adecuada por parte de un equipo de expertos que realizan una entrevista única (Gonzáles, 2011).
El objetivo del presente trabajo es evidenciar las ventajas de la cámara Gesell frente al método clásico de atención al niño que sufrió abuso.

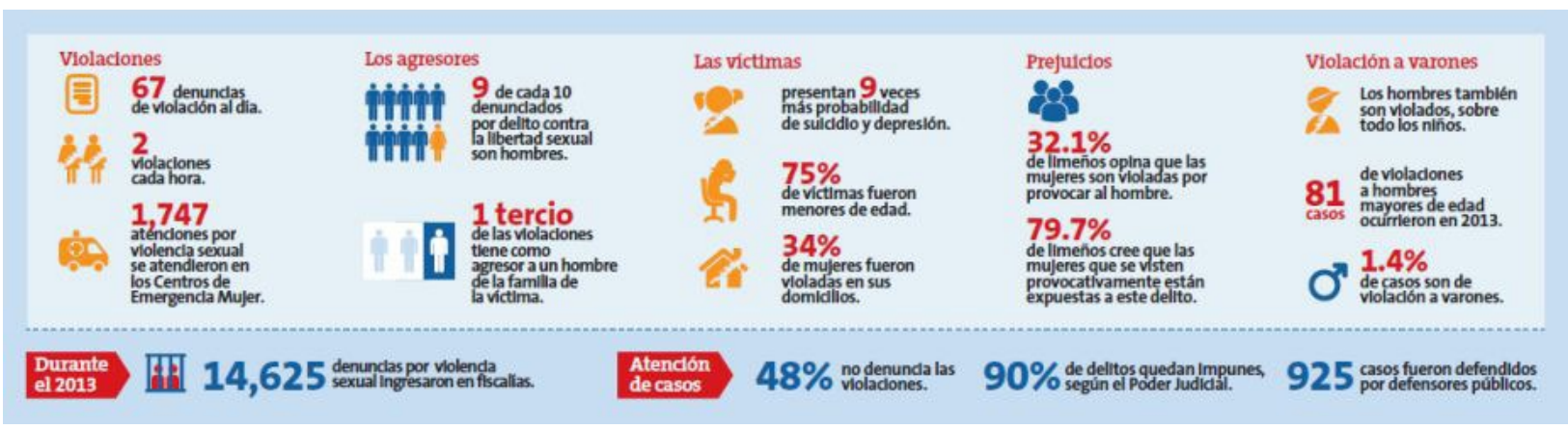

Figura 2. La violación sexual en Perú: Estadística de violaciones, agresores, víctimas y prejuicios y violación a varones. Fuente: http://peru21.pe/multimedia/imagen/t-252944

\section{Método y Materiales}

Es una investigación bibliográfica, realizada mediante el análisis documental. Se trabajó con información sobre la cámara Gesell y la revictimización en niños, niñas y adolescentes. Se examinaron distintas fuentes: aproximadamente 15 libros de leyes y normas, 10 manuales impresos, 50 artículos de revistas indizadas de las cuales se utilizó 20 por tener mayor información relacionada con el tema, y 5 bases de datos de ciencias sociales online, que son: CSIC, ISOC-Derecho, E-Journal, Clacso.Redalyc.org, Dialnet. Los descriptores utilizados en la búsqueda de la información fueron: revictimización, abuso sexual, cámara Gesell, uso de tecnologías en la revictimización, los cuales aportaron información sobre esta temática.

Tecnologías de la Información y Comunicación utilizadas en el Ámbito Jurídico

El impacto de las tecnologías de la información y de la comunicación (TICs) en el desarrollo de la sociedad actual es innegable, juega un rol transcendental que se manifiesta de forma transversal no solo en los aspectos cultural y social, sino también en lo económico y político. En la administración de justicia, la informática ofrece importantes soluciones operativas (Sanz, 1997). En primer lugar: la automatización de los sistemas de información judicial relativos a las fuentes de conocimiento jurídico (legislación, jurisprudencia y doctrina) puede dar respuesta a los problemas de hipertrofia e inflación normativa, característicos en la sociedad peruana (informática jurídica documental); en segundo lugar: la informatización puede aportar procedimientos dirigidos a la sustitución o reproducción de actividades del juez y también agilizar su trabajo (informática jurídica decisional); por último: la informática se proyecta sobre la propia organización operativa de las oficinas y dependencias judiciales (informática jurídica de gestión). Como puede verse, la informática forma parte de todas las instancias de la administración de justicia; se ha convertido en un soporte fundamental para agilizar el trabajo de jueces, especialistas, secretarios, abogados y litigantes.

El uso de las nuevas tecnologías en el sistema judicial puede hacer más eficiente y efectiva la gestión en los tribunales; reduce los costos del proceso, 
acorta los tiempos en la tramitación de las causas, agiliza las notificaciones judiciales y cualquier otra labor administrativa propia de la administración de justicia. Por su parte los abogados y litigantes pueden hacer un adecuado seguimiento de sus causas, tramitar electrónicamente recursos, asistir a teleaudiencias, brindar declaraciones y testimonios en forma virtual, etcétera. Los jueces acceden fácilmente a causas tramitadas en despachos distintos a los suyos, intercambian información con diferentes instituciones del Estado, emitir y notificar con rapidez resoluciones judiciales, grabar audiencias, entre otras (Lobos, 2010).

La implementación de tecnologías de la información y comunicación puede traer variados beneficios; pero su incorporación debe llevarse a cabo con las precauciones del caso. Las TIC en la administración de justicia pueden contribuir a que una mayor cantidad de personas accedan a la información judicial mejorando los estándares en materia de acceso a la justicia y la interacción entre las instituciones que conforman el sistema de justicia y los ciudadanos, como también mejorar la gestión de los tribunales. Los usuarios verán así satisfechas sus necesidades con mayor velocidad y eficiencia. Sin embargo, debe considerarse que la incorporación de nuevas tecnologías no debe convertirse en una barrera para quienes no cuenten con los recursos necesarios para llegar a tribunales. Su implementación en todo el sistema de justicia peruano es necesaria para garantizar la calidad del servicio y para que todos los peruanos sientan que el aparato estatal garantiza la seguridad jurídica de todos ellos.

\section{Abuso sexual en menores de edad}

Según Viviano (2012), el abuso sexual en menores de edad es el contacto e interacción entre una persona adulta con un menor de 18 ańos con la finalidad de obtener gratificación sexual y/o estimularse sexualmente él mismo o a otra persona. El abuso también puede ser cometido por una persona menor de edad, siempre y cuando medie una situación de abuso de poder por razón de edad, sexo, condición social, coerción, amenaza $\mathrm{u}$ otra situación que implique dominio o violencia. Se le llama "abuso" porque existe una relación de desigualdad entre quienes participan de esta interacción, encontrándose la persona abusadora en una posición de autoridad y poder que utiliza para someter al niño, niña o adolescente a las actividades sexuales. Esto sucede por una serie de aspectos que interaccionan entre sí y corresponden a los diversos niveles del desenvolvimiento humano establecidos en el modelo ecológico: personal, familiar, comunitario y social.

Como explica Barudy (1998), el abuso sexual produce en los afectados muchas consecuencias a corto plazo, como sentimientos de tristeza y desamparo, cambios bruscos de estado de ánimo, irritabilidad y rebeldía, temores diversos, vergüenza y culpa, ansiedad, bajo rendimiento escolar, dificultades de atención y concentración, desmotivación general, conductas agresivas, rechazo a figuras de autoridad, hostilidad y/o temor frente al agresor; a largo plazo, depresión manifiesta o enmascarada en diversos trastornos, distorsión del desarrollo sexual, intentos de suicidio o ideas suicidas, trastornos del aprendizaje, fuga del hogar, consumo de drogas y/o alcohol, delincuencia, interés excesivo por juegos sexuales, masturbación compulsiva y conductas sexuales de riesgo.

En nuestro medio podemos agregar las conductas presentadas en las llamadas Fiestas Semáforos o similares, en que las (los) adolescentes se autoexponen a peligro, asumiendo conductas sexuales impropias de su edade, como consecuencia de ello se producen embarazos adolescentes, abortos, enfermedades de transmisión sexual, fuga de hogares, deserción escolar, adicción a alcohol o drogas; que frustran el proyecto de vida de nuestra juventud.

Según las estadísticas de PNCVFS (Programa Nacional Contra la Violencia Familiar y Sexual), provenientes de la Encuesta sobre Abuso Sexual en niñas, niños y adolescentes de tres regiones del Perú en el 2004, para escolares de Primaria, en promedio, 1 de cada 10 escolares de primaria ha sufrido alguna forma de abuso sexual. Esta prevalencia en niñas fue de $13 \%$, mientras que en los niños de 7\%; el 62\% refirió ser afectado o afectada por gestos y miradas obscenas, el $40 \%$ por tocamientos o besos incómodos, el $28 \%$ manifestó que el abuso consistía en la obligación de 
realizar tocamientos a alguna persona y un $7 \%$, en relaciones sexuales forzadas (violación); como personas agresoras se señalan a: tíos, primos, padres, compañeros de colegio, profesores o profesoras, vecinos, desconocidos y empleadores. Para escolares de secundaria, se observa que las formas más frecuentes de abuso sexual son los tocamientos y/o besos incómodos, las propuestas indecentes, ser obligadas (os) a realizar tocamientos, intento de violación; con algo menos de incidencia aparece la violación; y finalmente, amenazas por no querer tener relaciones sexuales; las personas mencionadas como agresoras en el ámbito familiar fueron: el tío o la tía, seguido de los primos, en tercer lugar se encuentra el padre o padrastro, y en el cuarto lugar se encuentran los abuelos y hermanos. Lo más grave es que el ámbito familiar es donde ocurre con mayor frecuencia el abuso. Los agresores potenciales son personas cercanas a las víctimas. El enemigo está en casa gozando de la confianza de la familia, conviviendo con la víctima, a la espera del momento propicio para atacar y también para asegurarse de que esta no hable y el delito quede impune.

\section{La revictimización}

Para entender que es la revictimización, primero es necesario definir el término "víctima". Por víctima se entiende a "quien es objeto de un sacrifiาcio", o sea, quien se transforma en mero objeto a mano de otros, quienes la utilizan para su propia satisfacción (Unda, 2013). Tratándose de niños, niñas y adolescentes que han sufrido algún tipo de abuso sexual y que, en razón de su edad y condición, son afectados en su integridad física y/o psíquica y, por ende, en su construc $\neg$ ción subjetiva. Smith (2007) señala que el término "víctima" hace referencia a la personalidad del individuo o colectivo de personas en tanto es afectada por las consecuencias sociales de su sufrimiento, determinado por diversos factores físicos, psíquicos, sociales y políticos; así como por el medio ambiente o la técnica. La víctima es un tipo determinado de personalidad, de naturaleza depresiva, con un núcleo de angustia exógeno, motivado por factores de muy diversa naturaleza, sean estos físicos, socioambientales o psíquicos.
Para Castillo (2004), "revictimización” se puede precisar como la reitera $\neg$ ción de una victimización, según lo indica la palabra, y que apunta a la repro $\neg$ ducción de una situación de victimización anterior. Por tanto, la revictimización es una repetición de violencia contra quien ha sido previamente víctima de alguna agresión. Sin embargo, la palabra ha adquirido un sentido algo diferente; sirve para referirse en especial a las vivencias de maltrato sufridas por los niños y sus familiares, en el curso de intervenciones institucionales después de la denuncia de un abuso sexual u otra violencia; remite a un fallo en el abordaje y tratamiento de la situación de violencia.

Cualquiera que fuera el sentido de la palabra victimización, la víctima es el sujeto pasivo del estupro. El agresor la utiliza y somete antojadizamente, sin el más mínimo remordimiento. Cuando un niño, niña o adolescente es objeto de una nueva agresión, experimenta un doble maltrato; se repite el sufrimiento, el daño causado le produce heridas psicológicas profundas, que lo marcan para toda su vida. En la perspectiva mental del agresor, la víctima es un simple objeto a través del cual consigue el placer sexual.

La Cámara Gesell como tecnología que evita la revictimización

García (2012) menciona que en el año 2011, la Universidad de Yale conmemoraba el centenario de la fundación de su Child Study Center; que fue fundado por el prestigioso psicólogo y médico estadounidense Arnold Gesell, un apasionado psicólogo y pediatra especializado en el estudio del desarrollo infantil. Gesell dedicó su vida a observar a cientos de infantes, mientras desarrollaba un método que denominó "cinemanálisis. Su trabajo relacionado con el establecimiento de unas pautas de conducta infantil a lo largo del desarrollo está considerado como uno de los más influyentes en la puericultura de los años 40 y 50 , y que ha sido extrapolado al ámbito jurídico al haberse apropiado de esta tecnología en la actualidad.

El Ministerio Público menciona que la cámara Gesell fue diseñada y utilizada por el psicólogo y pediatra Dr. Arnold Gesell con la finalidad de observar el desarrollo y conducta de niños, para que 
estos no se sintieran presionados por la mirada de un observador. En atención al interés del niño, se muestra un evidente avance en la investigación sobre delitos sexuales cometidos contra menores de edad a través del uso de la Cámara Gesell, importante herramienta forense que evita la revictimización o un nuevo maltrato psicológico de los infantes durante el interrogatorio para esclarecer el caso (Peláez 2010).

Además se debe mencionar que el Art. 143 del Código de Procedimientos Penales señala que la declaración de la víctima será la que rinda ante el Fiscal de Familia, con arreglo a lo dispuesto en el Código de los Niños y Adolescentes; la Guía de Procedimiento para la Entrevista Única señala Que deben estar presentes el Fiscal Penal, el Fiscal de Familia, el efectivo policial (si la investigación está a ese nivel) y los abogados de la víctima y del presunto autor, ello a fin de no perjudicar el derecho de defensa del procesado. Se puede citar a la víctima cuando es mayor de 14 años y el mismo art $143^{\circ}$ señala que la confrontación procede a solicitud de la víctima.

La sala de entrevista-cámara de Gesell consta de dos ambientes dotados de alta tecnología y separados por un vidrio espejado que permite en- trevistar a un niño, niña o adolescente en condiciones adecuadas. Asimismo, permite grabar la entrevista en algún medio audiovisual que será posteriormente usado como prueba en el proceso judicial, sin la necesidad de que el menor de edad tenga que concurrir a los Tribunales dado a que su voz e imagen quedan perennizadas en la entrevista realizada con participación de un equipo de expertos dirigidos por el Fiscal de Familia y la presencia de los operadores de justicia llamados según ley. Desde la habitación contigua, estos agentes observan la entrevista que realiza el entrevistador a la víctima en el ambiente especialmente acondicionado para ello (Gonzales, 2011).

La cámara Gesell se convierte en el ambiente físico ideal para entrevistar al menor que fue víctima de una violación $y$, con la presencia invisible (para el menor) de todos los actores del proceso judicial, alcanzar el conocimiento necesario de los hechos que deben decidirse en el tribunal, sin causar al niño, niña o adolescente, ningún daño adicional al que ya padeció. Esta sala de entrevista que cuenta con herramientas de audio y vídeo permite perennizar las declaraciones del menor y las emociones que exteriorice mientras es entrevistado por el especialista designado por la autoridad judicial correspondiente.

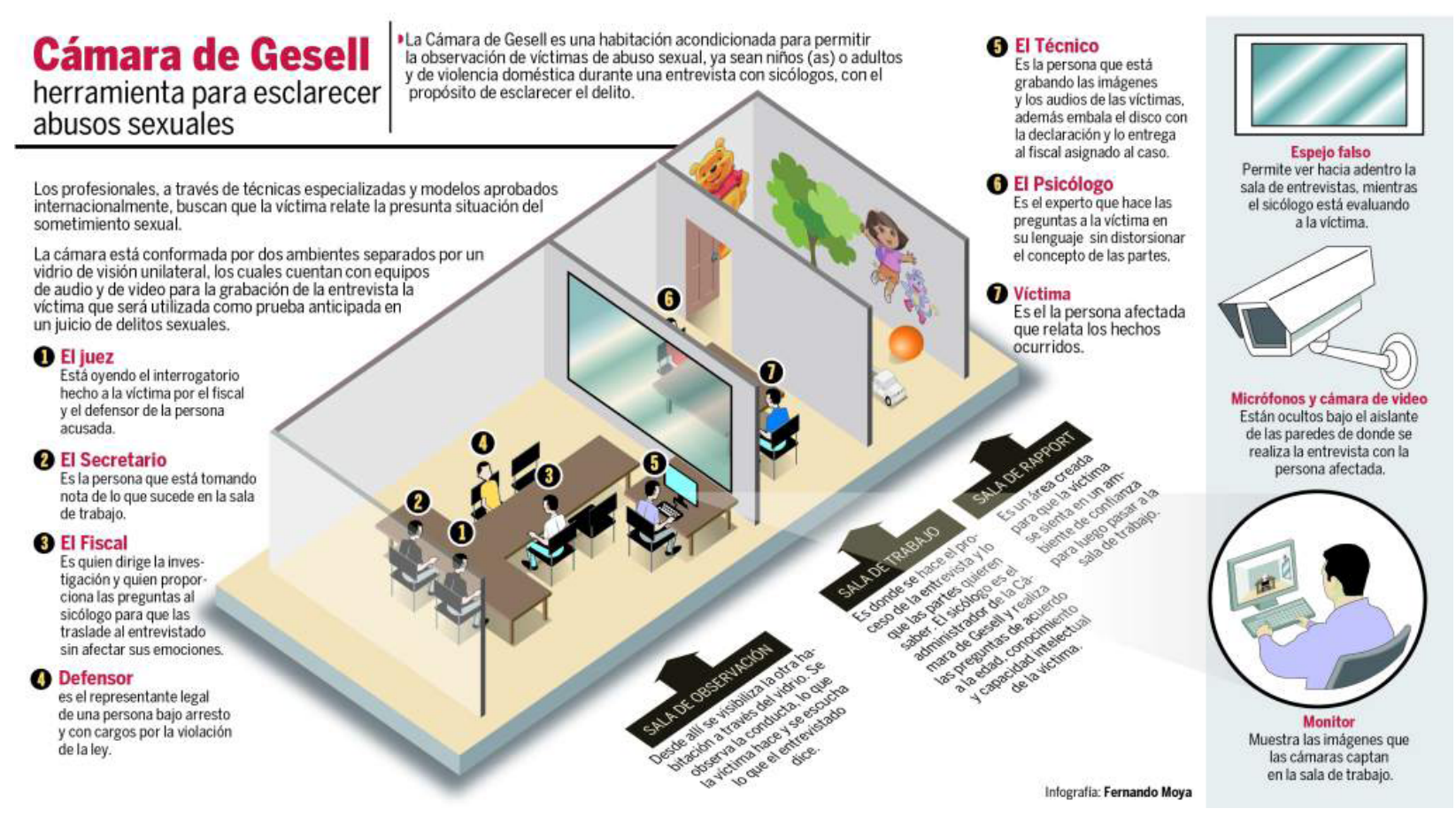

Figura 3. La Cámara de Gesell: herramienta que esclarece casos de abuso sexual.

Fuente: http://www.laprensa.hn/sucesos/752847-410/habilitan-en-san-pedro-sula-moderna-c\%C3\%A1 mara-de-gesell 
El apoyo de esta herramienta tecnológica que se comenzó a utilizar en Perú desde el año 2008 ha conllevado a que el ente jurídico estableciera normas y pautas para su uso y aplicación para evitar la revictimización en menores de edad, lo cual conllevó a que se aprobara con Resolución de la Fiscalía de la Nación N 1247 del 2012 la "Guía de procedimiento para la entrevista única de niños, niñas y adolescentes víctimas de abuso sexual, explotación sexual y trata con fines de explotación sexual", en la cual se norma los procedimientos a seguir en el uso de la Cámara Gesell, detallando la función y actuación de los intervinientes en la sala de entrevista única y los procedimientos a seguir dentro de la entrevista única, esta es entendida como un proceso reservado que busca evitar la revictimización. Peláez (2010).

\section{Ventajas de la Cámara Gesell}

Según Bravo (2011), son muchas las ventajas o beneficios que tiene la cámara Gesell, en comparación con la atención diaria al afectado:
- Es un ambiente de entrevista adecuado

- La noticia del hecho es informada a la Fiscalía de manera inmediata,

- Se crea un registro especial para las víctimas de violencia sexual que tiene carácter de reservado y en el que se cautela la identidad del NNA,

- Se realiza una sola entrevista a cargo de un profesional especializado,

- El Fiscal, la Policía y un profesional especializado desarrollan un cuestionario de evaluación dirigido al caso y donde se considera el objetivo de la investigación, con la finalidad de evitar los interrogatorios sucesivos,

- La Fiscalía, en coordinación con el Instituto de Medicina Legal, solicitará las pericias;

- La relación interinstitucional entre el Ministerio Publico, la Policía Nacional y el Ministerio de Justicia es más eficiente.

Todo esto hace que el trabajo sea más eficiente y ayuda a que el niño no padezca nuevos abusos y reciba adecuadamente la terapia que debería ser indicada por el especialista.

\begin{tabular}{l|l|l}
\hline REGISTRO DE TESTIMONIO \\
\hline
\end{tabular}

\section{La cámara Gesell por dentro}

Esta es la instalación acondicionada por el Ministerio Público para entrevistas únicas a menores victimas de violencia sexual.

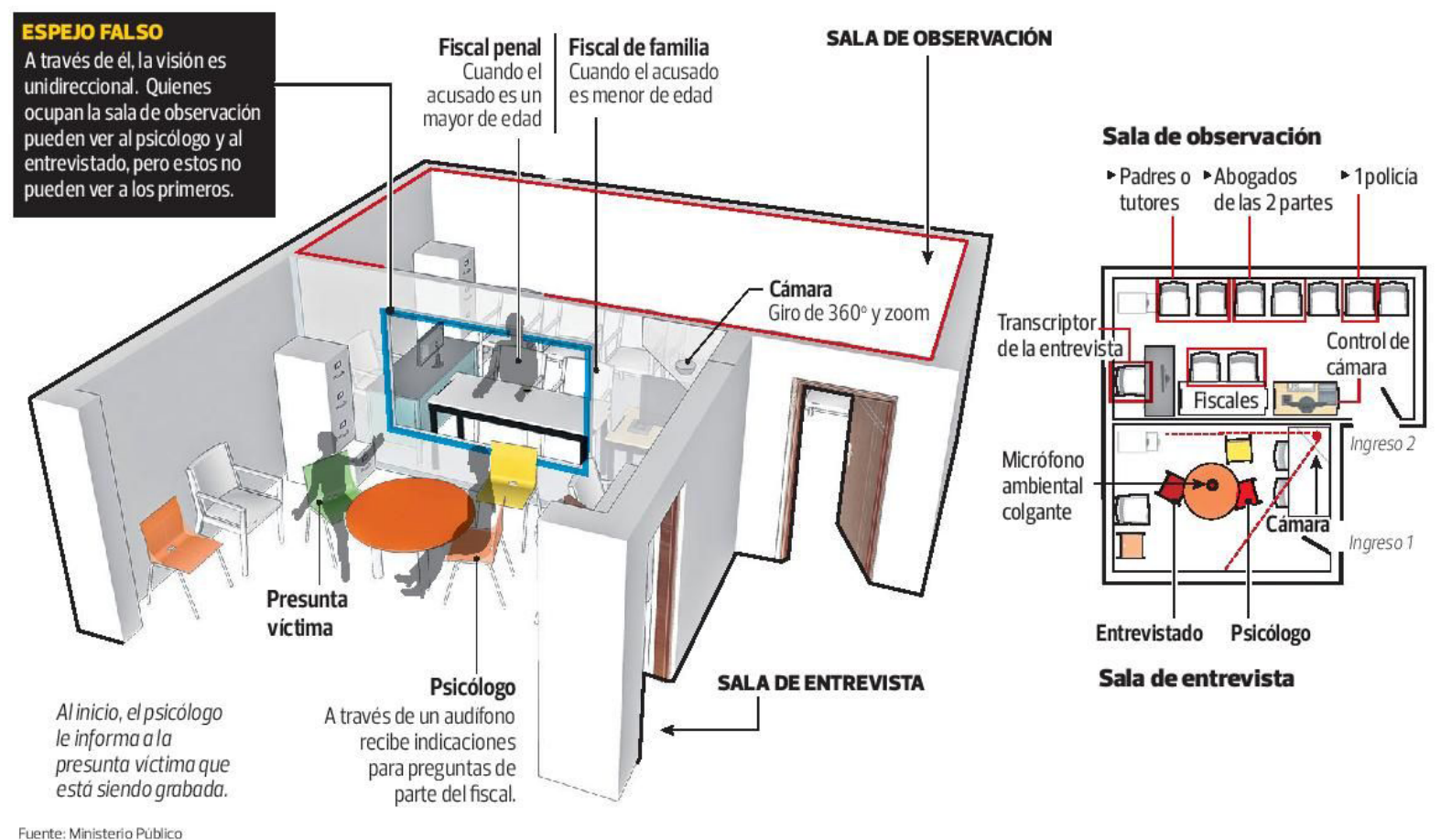

Figura 4. La camara de Gesell por dentro en el Ministerio Público.

Fuente: http://elcomercio.pe/visor/1858046/1251395-escolares-que-habrian-violado-ninos-pasaran-camara-gesell-noticia 
¿Cómo disminuir la revictimización de los niños, nińas y adolescentes que fueron objeto de violación sexual?

Para disminuir la revictimización de niños, niñas y adolescentes es necesario que el proceso penal se desarrolle y concluya con prontitud, atendiendo al Principio del Interés Superior del Niño; las diligencias judiciales deben realizarse con absoluta privacidad y contando con el auxilio de peritos especializados en la materia; el nińo, niña o adolescente debe ser informado sobre el motivo de las diligencias en que participa; se formularán preguntas relacionadas con la materia investigada, que sean claras, directas, de estructura sencilla a las que el niño, niña o adolescente pueda responder sin dificultad; la entrevista debe efectuarse en un lugar cómodo, seguro y privado (cámara Gesell), con presencia de profesionales especializados (trabajador social, psicólogo); el funcionario judicial debe evitar el contacto directo con la víctima; se deben acondicionar espacios físicos apropiados para tal fin; la declaración del niño, niña o adolescente debe ser la primera en recibirse; debe protegerse la identidad del menor, prohibiéndose cualquier tipo de exposición o publicación de su imagen; se protegerá la confidencialidad del niño, niña o adolescente registrando solo las iniciales de su nombre y apellido; la víctima debe dar su consentimiento para pasar los exámenes de ley; deben realizarse solo los peritajes corporales que resulten necesarios para averiguar la verdad. Estos se harán en compañía de un familiar. (Castillo, 2004)

En muchos países ya se está aplicando el uso de la cámara Gesell, como ejemplo se encuentra Guatemala, desde la instalación en el 2009 por la Cámara Penal de la Corte Suprema de Justicia de ese país, a pesar que la implementación de esta tecnología en un inicio tuvo obstáculos, con la petición del uso por el Ministerio Público se logró regular por el artículo 116 último párrafo del Decreto 5192, y que sirve como propósito para la reducción de victimización secundaria. (Gonzales, 2014) En el 2007, Republica Dominicana mediante la Resolución No 3687 - 2007, la Suprema Corte de Justicia de la República Dominicana, dispone la adopción de reglas mínimas de procedimiento para obtener las declaraciones de la persona menor de edad víctima, testigo en un proceso penal ordinario; en el artículo 3 se menciona que el interrogatorio se realizará con la Cámara de Gesell para cuando sean necesarias las declaraciones de una persona menor de edad, en calidad de víctima, testigo o coimputada, en un proceso seguido ante la jurisdicción penal ordinaria. En el 2012, mediante la Circular No 24 - 2012, la Corte Suprema de Justicia aprueba el Manual de Uso de Cámaras de Gessell, que tiene como objetivo la no revictimización de mujeres, personas menores de edad, personas adultas mayores, personas con discapacidad, que figuren como víctimas o testigos, en procesos judiciales, sin perjuicio de que sea utilizado en otros casos en que las circunstancias de la persona requiera este espacio para no ser revictimizada. (Guerrero, 2012)

\section{Conclusiones}

El abuso sexual a menores de edad en el mundo y en Latinoamérica está en incremento: El gobierno tiene que garantizar la defensa jurídica de esta población vulnerable, pues somos testigos que se está produciendo un aumento alarmante de denuncias por este tipo de abuso.

El proceso penal por violación sexual de niños, niñas y adolescentes es muy complejo, pues siempre está latente la posibilidad de revictimización del menor; sin embargo, es posible adoptar medidas para evitar daños colaterales a este menor. El Estado tiene la obligación de garantizar la seguridad jurídica de cada niño, niña o adolescente que fue víctima de estos delitos.

Una de las secuelas que se debe evitar es la revictimización en el niño violentado, es decir, que el niño abusado no debe volver a sufrir las consecuencias de la violencia de que fue objeto. La cámara Gesell puede ser una solución moderna y tecnológica para este problema. Tiene ventajas frente al método clásico que implica atender diariamente al niño afectado. La creación de un ambiente de entrevista adecuado para que el niño, nińa o adolescente se sienta cómodo al momento de su declaración; además, se realizará una sola entrevista a cargo de un profesional especializado, generalmente un psicólogo, de modo que el niño no tenga que reiterar su declaración y recordar todo el trauma padecido. 
El trabajo interinstitucional entre el Ministerio Publico, la Policía Nacional y el Ministerio de Justicia, permitirá que el proceso judicial sea más directo, se resuelva más rápido. Los psicólogos deben estimar qué terapia debe seguir el niño para lograr su recuperación.

La entrevista debe llevarse a cabo con participación de profesionales adecuadamente capacitados para evaluar al menor, siendo necesario que se cuente además con la participación de todo un equipo multidisciplinario, quienes, sin ser vistos por el menor, observen la entrevista a cargo del profesional designado y evalúen las condiciones en que el nińo, niña o adolescente se encuentra como consecuencia de la violencia sexual padecida. De esta manera el proceso se desarrollará garantizando la integridad emocional del menor.

La cámara Gesell implica la solución adecuada debido a su eficiencia socio-jurídica en el caso de violación sexual a niño, niñas y adolescentes. $\mathrm{Su}$ utilización en Perú debe dar respuesta a los constantes reportes de abuso sexual en menores de edad, evitando que durante la terapia que sigue para superar este abuso, vuelva a ser maltratada. Asimismo, resulta importante para evitar casos en que las víctimas son inducidas a retractarse de su inicial imputación, esto generalmente cuando el autor es un familiar cercano, causándole un daño aún mayor; pues ya su declaración habrá quedado perennizada, al igual que el daño psicológico sufrido a consecuencia de estos hechos.

\section{REFERENCIAS BibLiográficAS}

Gonzáles, M. (2011). El uso de la nueva tecnología en el acceso a la justicia de niños, nińas y adolescentes víctimas de violencia, abuso y explotación sexual infantil. (Tesis doctoral inédita). Universidad Nacional Mayor de San Marcos: Lima.

Sierra, G. (2013). Cámara de Gesell como herramienta investigativa en los abusos sexuales de niños y niñas. Caso de Honduras.

Paredes, J. (s.f.). La no revictimización de las víctimas de violencia sexual menores de edad en el nuevo modelo procesal penal mito o realidad. Recuperado de: http://www.teleley. com/articulos/art_010711.pdf

Peláez, J. (2010). Guía de Procedimiento para la Entrevista Única de Niños, Niñas y Adolescentes víctimas de abuso sexual, explotación sexual y trata con fines de explotación sexual. Ministerio Público.

Gonzáles Barbadillo, M. A. (2011). El uso de la nueva tecnología en el acceso a la justicia de niños, niñas y adolescentes víctimas de violencia, abuso y explotación sexual infantil.

Viviano, T. (2012). Abuso Sexual: Estadísticas para la reflexión y pautas para la prevención. Programa Nacional contra la Violencia Familiar y Sexual.

Sanz, F. J. (1997). El Derecho ante las nuevas tecnologías de la Información.

Barudy, J. (1998). El dolor invisible de la infancia: Una lectura ecosistémica del maltrato infantil. Barcelona: Paidós.

PNCVFS. (2004). Maltrato y abuso sexual en niños, niñas y adolescentes: Estudio realizado en San Martín de Porres, Cusco e Iquitos. Lima: MIMDES.

Bravo, E. D. (2011). La implementación de la cámara de Gesell como medio Alternativo para la no revictimización en el proceso Penal ecuatoriano. (Tesis doctoral, Universidad Internacional SEK).

Lobos R. (2010). El Uso de Nuevas Tecnologías en el Sistema Judicial: experiencias y precauciones.

Unda, N., \& Dupret, M. A. (2013). Revictimización de niños y adolescentes tras denuncia de abuso sexual/Re-victimization of children and adolescents after reporting sexual abuse.

Smith, B., \& Alvarez, M. (2007). Revictimización un fenómeno invisibilizado en las instituciones. Rev. Med. leg. Costa Rica, 24(1), 65-100.

García, E. S. (2012). Muybridge y Gesell: Pioneros de los métodos de investigación visual en Psicología. Revista Costarricense de Psicología, 31(1), 191-210.

Catillo S. (2004). Directrices para reducir la revictimización de las personas menores de edad en procesos penales. Costa Rica: CONAMAJ- UNICEF.

Zanetta, M. (2006). La cámara Gesell en la investigación de delitos sexuales. Revista electrónica mensual de derechos existenciales, 55 (7) 1-17. Recuperado de http://www.revistapersona.com.ar/Persona55/55Zanetta.htm

Resolución de la Fiscalía de la Nación No. 1247

http://www.munizlaw.com/normas/2012/Mayo/23-05-12/ Res.\%20N\%C2\%BA\%201247-2012-MP-FN.pdf

Bagnasco, M. Gelso, E. \& Guadalupe, M. (2015). Guía de abordaje integral ante situaciones de Violencia Sexual hacia Niños, Niñas y Adolescentes. Ministerio de Salud - Provincia de Buenos Aires.

González, S. (2014). Adición de norma procesal penal para uso de la cámara gesell en casos de abuso sexual infantil. Tesis para optar el grado de licenciatura en ciencias jurídicas y sociales.

Guerrero, R. (2012). Guía de uso de la Cámara Gesell. Dirección de Protección a Victimas, Testigos y Miembros del Ministerio Público de Bolivia. 